ENCYCLOPEEDIE Encyclopédie berbère

BERBERE

9 | 1991

9 | Baal - Ben Yasla

\title{
Bélier à sphéroïde
}

(Gravure rupestre de l'Afrique du Nord)

G. Camps

\section{OpenEdition}

\section{Journals}

Édition électronique

URL : http://journals.openedition.org/encyclopedieberbere/1511

DOI : 10.4000/encyclopedieberbere.1511

ISSN : 2262-7197

\section{Éditeur}

Peeters Publishers

\section{Édition imprimée}

Date de publication : 1 avril 1991

Pagination : 1417-1433

ISBN : 2-85744-509-1

ISSN : $1015-7344$

\section{Référence électronique}

G. Camps, « Bélier à sphéroïde », Encyclopédie berbère [En ligne], 9 | 1991, document B54, mis en ligne le 01 avril 2013, consulté le 25 septembre 2020. URL : http://journals.openedition.org/ encyclopedieberbere/1511; DOI : https://doi.org/10.4000/encyclopedieberbere.1511

Ce document a été généré automatiquement le 25 septembre 2020.

(c) Tous droits réservés 


\section{Bélier à sphéroïde}

\section{(Gravure rupestre de l'Afrique du Nord)}

\section{G. Camps}

Parmi les très nombreuses gravures rupestres de l'Atlas il est un thème qui a très tôt attiré l'attention des archéologues, celui d'un ovidé paré d'une coiffure globuleuse de forme sphérique et de divers attributs, l'ensemble étant communément désigné sous l'appellation de « bélier à sphéroïde ».

Comparaison proposée par E.F. Gautier entre une représentation du dieu Amon-Ra de Karnak (Égypte) et un ovin d'une gravure rupestre de Zénaga (Algérie).
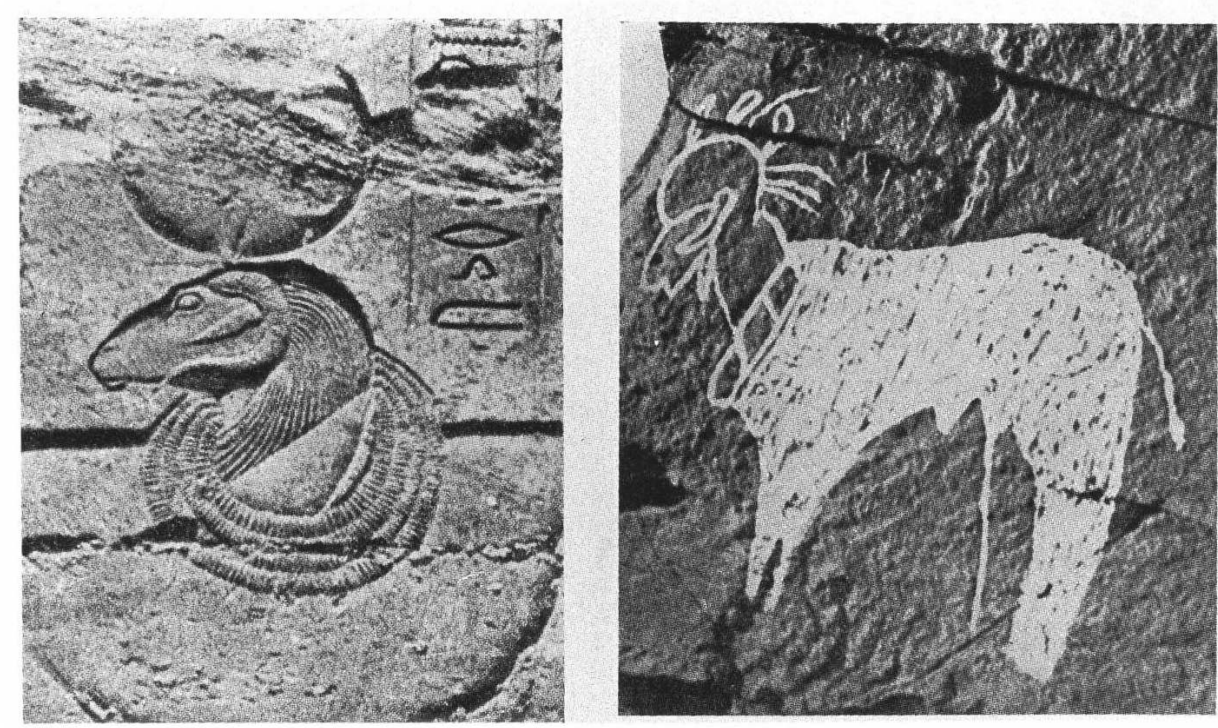

2 Ces curieuses figurations parmi lesquelles se comptent quelques chefs-d'œuvre de l'art rupestre nord-africain ont donné lieu à de nombreux commentaires et hypothèses.

3 Les plus anciens sont fondés sur une analogie de ces représentations avec celles du dieu égyptien Amon-Râ. Le dieu de Thèbes, Amon, était figuré depuis l'ancien Empire sous la forme d'un bélier ; par suite de sa fusion avec Râ, le dieu solaire, il faut affublé, surtout 
devant le Nouvel Empire, du disque solaire. Or les coiffures sphériques des ovins de l'Atlas sont représentées à plat sur deux dimensions et paraissent être des disques. La confusion devenait d'autant plus facile que parmi les attributs qui s'échappent des sphéroïdes sur de rares gravures, les plus soignées (Bou Alem, Aïn Naga, Zénaga) certains de forme courbe et redressée sont assez semblables aux uraei (serpents dressés qui flanquent habituellement le disque solaire égyptien). Les premiers spécialistes de l'art rupestre nord-africain étaient donc conduits à voir dans les représentations atlasiques des figurations d'une divinité africaine d'origine égyptienne.

4 Cette opinion était confortée par l'importance donnée alors au culte du dieu Amon durant l'Antiquité classique. On admettait facilement qu'à la tête du panthéon africain siégeait un dieu suprême, Amon ou Ammon*, devenu célèbre dans le monde grec dès le $\mathrm{VI}^{\mathrm{e}}$ siècle av. J.-C. par son oracle de l'oasis de Siouah. Les auteurs croyaient que ce dieu oraculaire, confondu avec le dieu thébain Amon-Râ, avait étendu son influence jusqu'à l'extrême ouest de l'Atlas. D'autre analogies, de nom cette fois, permettaient d'établir une autre équivalence: on savait qu'à Carthage le dieu Baal-Hammon (dont le nom semble signifier en phénicien le Maître des Brûle-parfum) détenait un rôle prééminent ; les Africains n'auraient-ils pas confondu ce dieu punique et le dieu égyptien sous le même nom ? Enfin, dernier élément à l'appui de cette thèse, R. Basset retrouvait chez les Guanches des Iles Canaries, le nom d'Aman qui signifiait "Seigneur » et était appliqué au Soleil.

Amon dieu-bélier primordial, devenu dieu solaire par sa fusion avec Râ aurait ainsi établi de proche en proche sa domination sur les panthéons inorganisés des Barbares de l'Ouest africain, mais ceux-ci restés à un stade plus primitif adoraient le même dieu sous sa forme animale. Ce culte aurait subsisté très tard puisque El Békri, au XI ${ }^{\mathrm{e}}$ siècle, signalait l'existence d'un culte du bélier dans le Sous. 


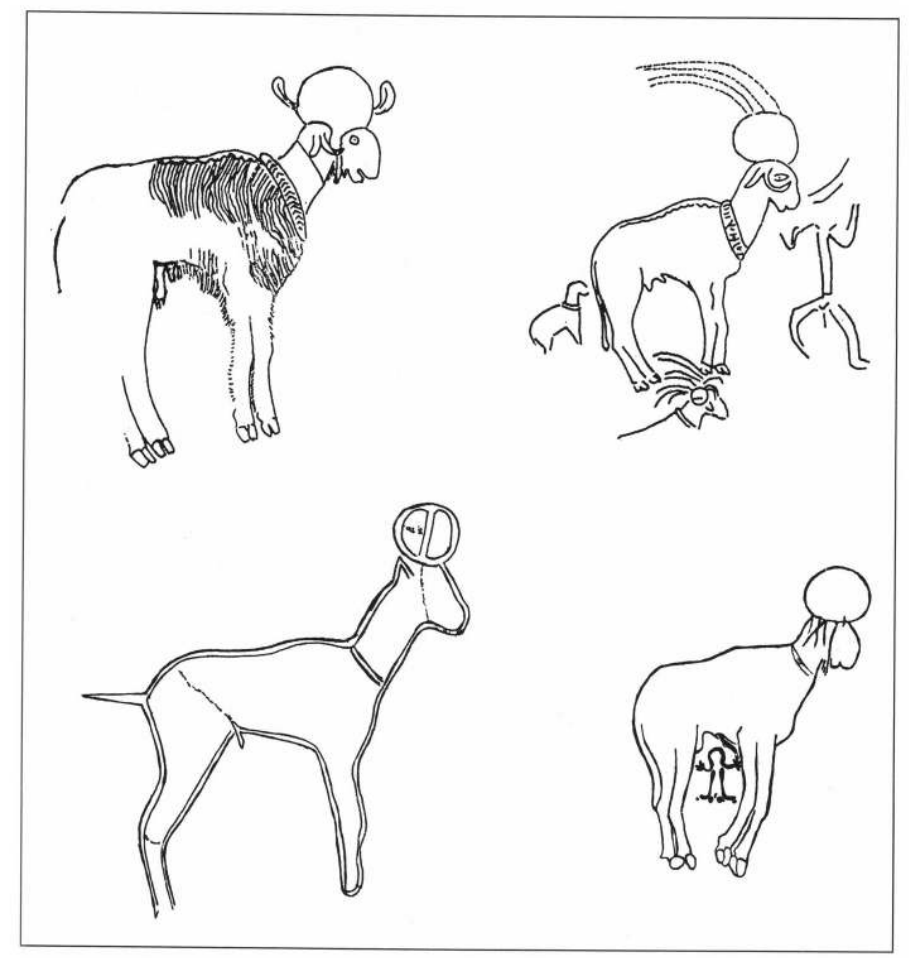

1. Bou Alem, 2. Guelmouz el-Abiod, 3. Dayet es-Stel, 4. Hadjra Sidi Bou Baker.

6 La thèse était trop simple. La coiffure des béliers de l'Atlas n'est pas un disque solaire et les représentations égyptiennes, situées arbitrairement à l'origine de celles de l'Atlas leur sont postérieures de 2 à 3 millénaires puisque celles-ci appartiennent au Néolithique ancien. St. Gsell, qui dans le tome 1 de son Histoire ancienne de l'Afrique $d u$ Nord, avait admis la thèse égyptienne, la rejetait définitivement en 1927, dans son tome 5. Dès 1900, G.B.M. Flamand avait émis une hypothèse inverse, et plus vraisemblable, celle d'une influence libyenne sur le culte du bélier de Mendès. Ce qui n'empêchait pas F. Benoît, en 1930, encore, de parler de béliers porteurs de disque solaire flanqué de deux uraei, mais en même temps il établissait une comparaison avec les parures de béliers que les tribus Songhaï sacrifiaient sur les bords du Niger. Ces parures sont des calebasses munies de banderoles de cuir. Le disque solaire devenait une simple calebasse comme l'avait déjà suggéré G.B.M. Flamand en 1900.

7 Se fondant sur la présence de cette calebasse et de colliers portés par certains ovins, L. Joleaud présenta une thèse nouvelle assez surprenante qui associait plusieurs thèmes des gravures rupestres à un rite en vue d'obtenir la pluie. Cette hypothèse était appuyée sur des arguments spéciaux (ainsi tout trait figuré sous les animaux était considéré comme une émission d'urine ou de semence, même lorsque ce trait jaillissait, comme à Zénaga, des mamelles d'une brebis), et sur des documents mal interprétés, comme les mouflons de Kef Fentéria qualifiés de moutons à sphéroïde. Elle ne rencontra guère d'écho favorable.

8 Le dernier commentaire des gravures rupestres du Sud oranais relatives à ces ovins a été donné par H. Lhote qui fait connaître de nouvelles représentations dans les monts des Ksour et le Djebel Amour et les monts des Ouled Naïl. La seule idée nouvelle exprimée et qui surprend est que les moutons représentés dans les gravures qui 
appartiennent au style naturaliste jugé le plus ancien, même ceux munis de collier et de coiffure sphérique, ne sont pas des animaux domestiques. Nous allons successivement tenter de reconnaître l'espèce représentée, d'examiner les attributs qui ornent ces animaux, enfin de définir les rapports entre les figures animales et les figures humaines qui leur sont associées.

\section{L'espèce représentée}

9 L'animal qui figure plus d'une soixantaine de fois, identifié par son sphéroïde, est très différent du mouton qui vit aujourd'hui dans les mêmes régions. Cependant la répétition de ce qui pourrait paraître une anomalie est trop constante pour qu'on puisse l'attribuer à la fantaisie des artistes. Si on examine les meilleures représentations, comme celles de Bou Alem, Aïn Naga, Hadjar Sidi Bou Beker, on remarque que ces animaux ont un corps étroit porté par des membres longs et secs, la croupe toujours plate, parfois anguleuse, aux gigots plats. La queue, mince et longue, descend jusqu'à l'articulation métatarsienne, mais dans certaines figures (Daïet es-Stel, Enfous) elle est plus large et s'arrête au bas du gigot.

10 La tête est tout à fait remarquable, elle est, dans les meilleures œuvres, plus courte, voire camuse, que dans la réalité. Les ressemblances entre les têtes d'Aïn Naga, Bou Alem, Gada el-Kharrouba, Guelmouz el-Abiod, Hadjerat Sidi Bou Beker, Daïet es-Stel, etc. sont telles qu'il s'agit manifestement d'une donnée stylistique qui aurait voulu accentuer la convexité du chanfrein et le raccourcissement du museau. Or dans la plupart des cas il s'agit d'un bélier dont la tête, dans la nature, est effectivement plus convexe que celle des brebis. Précisément la brebis aux mamelles gonflées de Zénaga a une tête bien plus fine et une convexité moins marquée ; toutefois une brebis de Bou Alem a exactement le même profil que le mâle du même site.

Béliers à poils ras du Sahara méridional : mouton maure du Mali et mouton targui de la variété AraAra du Niger.

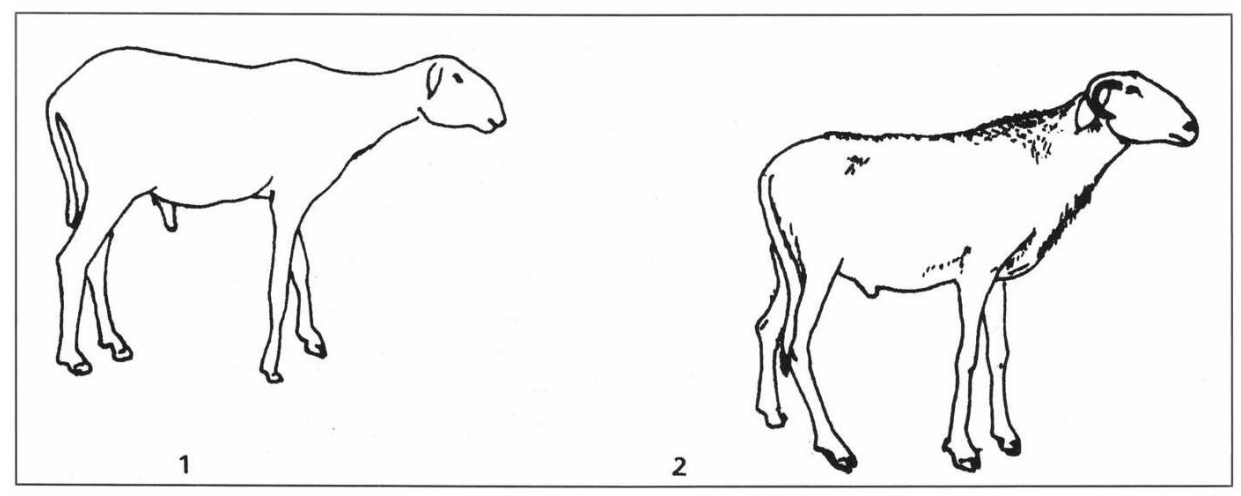

11 Qu'ils soient mâle ou femelle ces animaux portent des cornes qui sont encore figurées dans les représentations moins réalistes ou d'un schématisme avancé (El Richa, Safiet Bou R'Nam, Oued Nourème), or la forme et la taille de la seule corne visible sur le profil de ces ovins est d'une constante remarquable, elle est toujours petite, grêle, à simple courbure, incurvée vers l'arrière pour revenir vers l'avant au-dessous de l'œil, sauf à Khrelouat Sid Cheikh où elle revient au-dessus de l'œil. Il s'agit dans ce cas, peut-être, 
d'une tentative ou d'un début de déformation volontaire qui, comme nous le verrons, semble avoir été parfois pratiquée par les bergers de l'Atlas à cette époque.

L'oreille, qui est plus facilement omise que la corne, est pendante, assez longue; sa racine est cachée par le départ de la corne.

Les détails de la robe des animaux ne sont que rarement indiqués. Dans les œuvres les plus soignées (Aïn Naga, Safiet Bou R'Nam, Sidi Bou Beker, Guelmouz el-Abiod...) la partie endopérigraphique est entièrement polie et peut avoir été peinte. L'un des ovins asexué de Zénaga a le corps entièrement piqueté à l'exception du collier et de la tête ce qui révèle bien l'intention de figurer le pelage. Le même figuré apparaît sur le corps d'un mâle de Khrelouat Sid Cheikh, tandis qu'à Hadjar Berrik, à Dehar Bel Haadi et ailleurs, le corps de l'ovin très stylisé est, suivant une convention fréquente dans le style dit décadent, cloisonné, sans doute pour suggérer des variations de couleur dans la robe. Le beau bélier de Bou Alem est particulièrement détaillé ; sur le poitrail, le garrot et l'avant-main, des poils assez longs sont figurés par des stries ondulées du plus bel effet; l'arrière du corps, comme dans la nature, a un pelage moins fourni. La figuration de ce pelage avait fait penser, un moment, que l'animal figuré était un bouc (S. Gsell, 1901).

En fait, l'ensemble des caractères fidèlement reproduits sur les meilleurs gravures permet de reconnaître des moutons, mâles et femelles, appartenant à une ou plusieurs variétés du mouton à poil de l'espèce Ovis longipes Fitzinger. Ce mouton, qui a disparu du Maghreb et du nord du Sahara, subsiste, sans grand changement, dans le Sahel, depuis la Maurétanie jusqu'au Tchad (Ovis sodonica Sanson). C'est l'actuelle race targui de l'extrême Sud algérien, du Mali et du Niger, qui présente le plus de traits communs avec la variété la plus fréquemment représentée dans l'Atlas. G. Doutresoulle (1952) reconnaît au grand mouton targui d'une taille de $0,70 \mathrm{~m}-0,80 \mathrm{~m}$, un garrot saillant, un front bombé, des oreilles tombantes, des cornes en larges spires, une crinière fréquente chez le mâle, une queue longue et mince descendant jusqu'au jarret. Comme on le voit, cette description s'applique à la lettre aux ovins des gravures de l'Atlas. Le même auteur distingue au Niger, parmi les moutons à poils ras, deux variétés, le Bali-Bali qui est élevé chez les sédentaires et le Ara-Ara qui est proprement touareg, aux cornes moyennes, incurvées en arrière, haut de 0,60 à $0,70 \mathrm{~m}$, dont le corps étroit repose sur des membres secs et minces, les gigots sont plats. Enfin G. Curasson dans sa monographie sur le mouton du Soudan français signalait, parmi les moutons maures, le Touabir, plus petit et plus court que le mouton targui mais dont les poils, qui atteignent 8 à $10 \mathrm{~cm}$, sont assez longs pour être tissés. C'est de cette variété que se rapproche le bélier de Bou Alem, qui est plus ramassé que les autres et dont les longs poils sont indiqués d'une manière très réaliste.

15 Les pendeloques sont assez fréquentes chez les différents moutons à poils du Sahel. Ce détail anatomique ne semble avoir été indiqué que dans trois gravures d'ovins à sphéroïde, à Zénaga, à Daïet es-Stel et sur un jeune mâle de Bou Alem. Dans d'autres cas, comme à Khrelouat Sidi Cheikh, de prétendues pendeloques peuvent n'être que les extrémités des jugulaires de la coiffure.

Les béliers et les brebis coiffés et ornés de colliers que les artistes préhistoriques ont représentés appartiennent sans conteste aux mêmes variétés que les ovins à poil ras du Sahel. Ces derniers sont les représentants d'une très vieille race domestique que l'Égypte a connu jusqu'au Moyen Empire (Ovis palaegyptiaca Gaillard), cette variété dite aussi bélier de Mendès se distingue de celle de l'Atlas pour l'encornure dont les spires 
très lâches se développent horizontalement. Cette caractéristique subsiste cependant dans plusieurs variétés du Sahel; la race peul de Sambouran, en particulier, a des cornes dont le développement horizontal atteint une dimension égale à la hauteur de l'animal. Les cornes à spires lâches se retrouvent aussi chez certains béliers de la race targui. Parmi les gravures rupestres de l'Atlas, deux figurations se rapportent, peutêtre, à cette variété. L'une à Hadjar Berrik, l'autre à Kef Marbéah, dans le Constantinois. Dans cette dernière gravure $C$. et $\mathrm{L}$. Lefebvre proposent d'identifier les addax en raison de l'angle formé par les cornes dressées obliquement ; c'est très vraisemblable mais la longueur de la queue et l'aspect des cornes les rapprochent davantage des ovins que des hippotraginae. Effectivement, la forme des cornes des ruminants de Kef Marbéah présente de grandes analogies avec celles des moutons hongrois de la variété racka. Quoi qu'il en soit, aucune représentation d'Ovis palaegyptiaca n'est assurée dans l'Atlas.

Que conclure sinon que les moutons de l'Atlas sont, avec les autres moutons à poils de l'Égypte ancienne et du Sahel d'aujourd'hui, les représentants d'une très ancienne race domestique, certainement la première en Afrique.

18 Mais s'agit-il bien d'animaux domestiques sur nos gravures? La présence de parures aussi complexes que la coiffure globuleuse, le collier et parfois le "caparaçon ", pourrait servir de réponse immédiatement favorable à cette question. Or $\mathrm{H}$. Lhote s'élève contre cette opinion pourtant universellement partagée sous prétexte que le sphéroïde est parfois figuré sur d'autres animaux considérés comme sauvages. On cite le cas du grand buffle (Homoïoceras antiquus) de Trik el-Beïda, dans les monts des Ksour ; or il s'agit d'une figure très maladroite sur laquelle les cornes sont décalées; on peut même se demander si ce disque, fort petit et qui n'a rien de comparable par ses dimensions avec la coiffure des béliers, n'est pas la figuration schématique du toupet de poils parfois indiqué en arrière de l'encornure. On ne peut non plus retenir les disques plus ou moins douteux qu'on a cru voir sur des gravures négligées (oryx de Kef Bou Beker).

Un autre argument présenté par $\mathrm{H}$. Lhote à l'encontre de la domestication du mouton est tiré d'une scène de Rosfat el-Hamra Men el-That où un archer suivi de chiens est encadré par deux ovins; tous les sujets sont tournés vers la droite. Aucun élément ne permet d'affirmer qu'il s'agisse d'une chasse au mouton. Comme le fait remarquer F. Libmann, la flèche que s'apprête à décocher l'archer est parallèle au corps du premier mouton et ne le menace nullement, la cible est ailleurs. L'homme menacerait-il vraiment le mouton que je ne verrai pas pourquoi il s'agirait d'une "chasse »; on peut en effet sacrifier un animal domestique avec une flèche décochée à bout portant. Les Massaï saignent périodiquement leurs bœufs en perçant la veine jugulaire avec une flèche. 


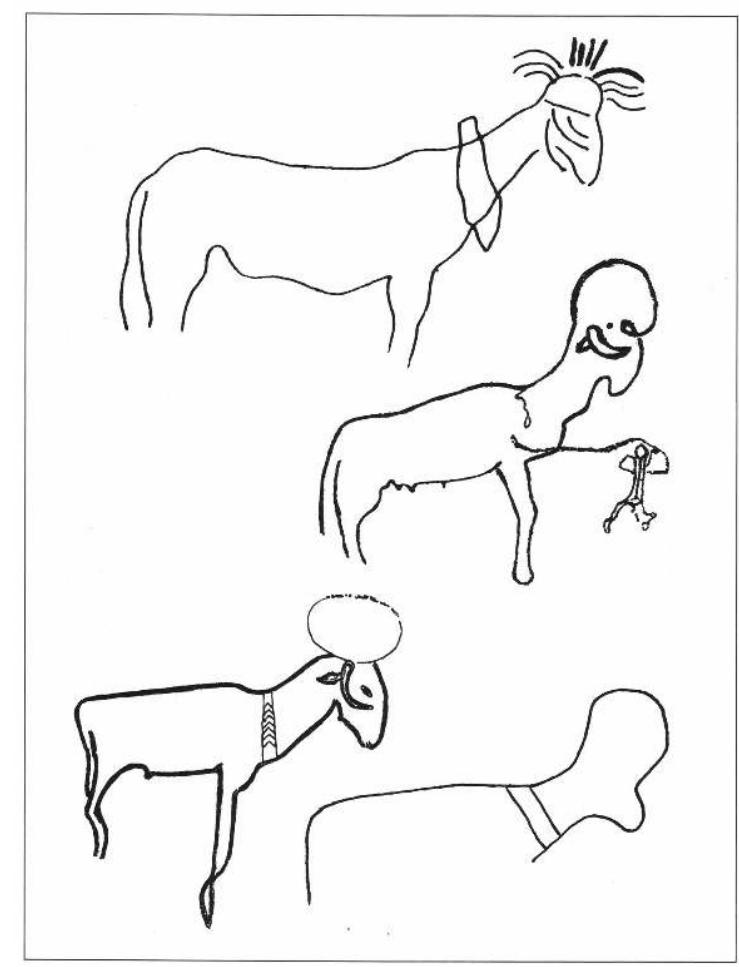

1. de Zénaga portant une calotte ornée de rameaux et d'un lourd collier d'épaule, 2. bélier d'El-Hasbaya à sphéroïde non rigide, le petit personnage a été rajouté, 3. bélier raté de la Dayet el-Hamra portant le sphéroïde et le collier tressé, 4. silhouette stylisée d'un bélier de Safiet bou Rhénan, la coiffure se confond avec la tête, mais le collier n'a pas été oublié.

Le troisième argument opposé à la domestication du mouton serait l'existence d'ovins sauvages au Maghreb durant les temps préhistoriques. Il s'agit d'une affirmation exagérée et sujette à caution. Les rares ossements attribués par R. Vaufrey à un ovin et trouvés dans des gisements capsiens de Redeyef, Bir Khanfous, Relilaï et Mechat elArbi, n'emportent nullement la conviction car, comme l'on fait remarquer G. Espérandieu et R. Vaufrey lui-même, ces ossements peuvent appartenir à de petites femelles de mouflon (Ammotragus lervia). Ni C. Arambourg, ni G. Espérandieu ne croyaient à l'existence d'un oviné nord-africain dont la niche écologique est occupée par le mouflon à manchette. Les ossements incriminés seraient-ils vraiment des restes de moutons qu'il ne prouveraient pas grand chose car les couches qui les ont livrés appartiennent au Capsien supérieur, c'est-à-dire qu'ils peuvent être précisément contemporains de l'introduction du mouton domestique sur les bords de la Méditerranée, à la fin du VII ${ }^{\mathrm{e}}$ millénaire.

21 Rappelons enfin que la souche du mouton ne peut être le mouflon à manchette (Ammotragus lervia) qui est d'un genre différent et dont le caryotype est distinct de celui du mouflon d'Orient (Ovis orientalis) qui est l'ancêtre du mouton domestique.

La cause est entendue: les moutons de l'Atlas sont des représentants d'Ovis longipes Fitz., d'origine exotique et sont donc des ovins domestiques, comme leurs congénères de l'Égypte néolithique et pharaonique et comme les actuels moutons à poil ras touaregs, maures et peuls. 


\section{Les attributs culturels}

Ces ovins, qui ne sont pas toujours des béliers, ont reçu une parure qu'il importe d'analyser avant de rechercher sa signification. Lorsqu'elle est complète, cette parure affecte la tête, le cou et l'échine. La parure de tête est la plus fréquente et paraît le minimum indispensable pour sacraliser l'animal. Rares en effet sont les animaux qui portent un collier sans avoir de sphéroïde ; on citera le petit ovin de Guelmouz el-Abiod qui suit les béliers à sphéroïde, mais comme nous le verrons les ovins de cette scène complexe ont reçu leur parure après coup. On peut citer aussi le petit ruminant indéterminé de l'Oued Dermel qui porte un lourd collier, l'allure générale n'est pas celle d'un ovin et les cornes redressées vers l'avant et le haut sont plutôt celles d'une gazelle, seule la queue longue et mince rappelle le mouton. Mis à part ces deux cas litigieux, je ne connais pas de mouton paré d'un collier qui ne soit coiffé du sphéroïde ou qui n'ait les cornes transformées en disque (El-Harara).

\section{Parure de tête, le « sphéroïde »}

Cette parure est la plus importante, la plus visible et la plus caractéristique. L'essentiel est constitué par un corps globuleux fixé à l'occiput de l'ovin. Il faut examiner en premier lieu les gravures les plus réalistes qui permettent de comprendre les représentations plus schématiques. Prenons comme exemples caractéristiques les béliers et brebis de Bou Alem, le bélier de la Gada el-Kharrouba, la belle tête d'Ain Naga, la brebis de Zénaga. Ces gravures reproduisent fidèlement la coiffure qui est sphérique. On a parlé de casque et de calebasse, mais si cette coiffure a une certaine rigidité il importe de remarquer qu'elle est munie de jugulaires qui descendent entre la corne et la joue et se terminent par de petites franges (Aïn Naga). Des gravures aussi détaillées que celles de Bou Alem ou Aïn Naga n'indiquent aucune marque de fixation de la jugulaire sur le "sphéroïde ", alors que les boutons du cache-sexe de l'homme d'Aïn Naga sont minutieusement notés. Ceci nous fait admettre que la jugulaire, en continuité avec la coiffe est en même matière qu'elle. Compte tenu de ce détail technique et de la rigidité du "sphéroïde ", nous sommes conduits à croire que l'ensemble était en cuir. Le découpage en festons des bords de la jugulaire (Bou Alem) et les franges des extrémités (Aïn Naga), confirment cette opinion. Le port redressé de la tête des ovins porteurs d'une telle coiffure sous-entend que celle-ci était suffisamment légère pour ne pas gêner l'animal; l'usage du cuir plus que d'une calebasse convient à l'attitude fièrement campée des béliers et brebis à sphéroïde. 


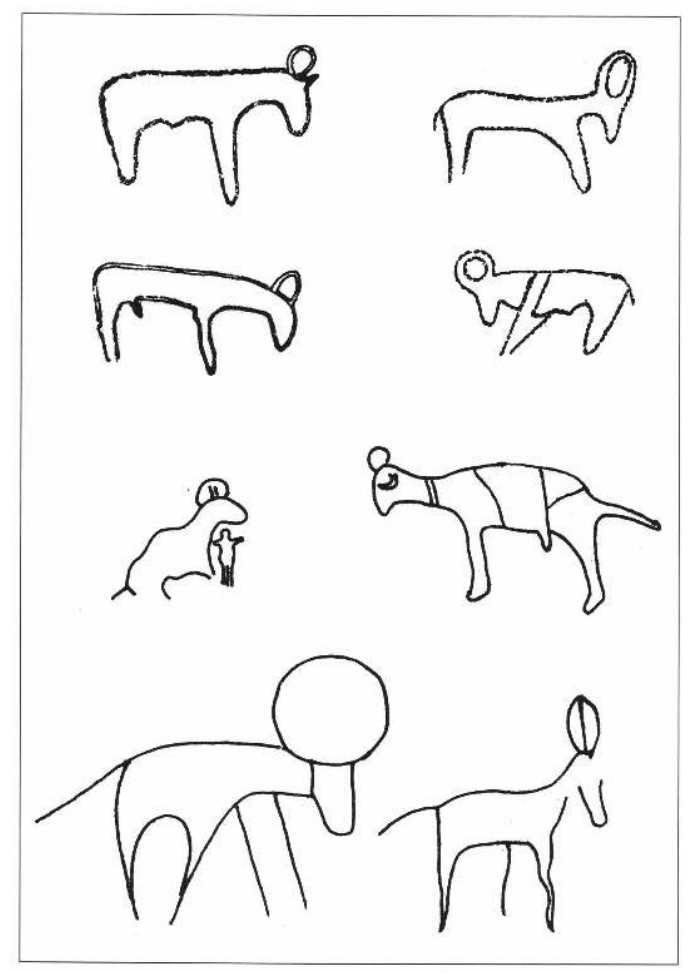

Cet attribut nous paraît donc être un bonnet de cuir, coiffure cérémonielle dont on dotait l'animal à certaines occasions. Or ce n'est pas sans surprise que l'on reconnaît une coiffure identique sur la tête des personnages de Khrelouat Sidi Cheikh et de Dekhilet el-Ateuch. Comme sur les béliers, ce bonnet est muni de jugulaires frangées et des rameaux rayonnant sont piqués dans cette coiffure. La position frontale du personnage dont le visage est vu de face est elle-même exceptionnelle dans le style archaïque naturaliste. Il s'agit peut-être d'une divinité à laquelle était associé le mouton porteur de la même coiffure. A Moghrar Tahtani, un petit personnage, très stylisé, en position d'orant, porte cependant une coiffure comparable qui ne doit pas être confondue avec les plumes ou autres ornements de tête si fréquents dans le représentations rupestres nord-africaines.

Le bonnet est la parure de tête principale; il semble avoir suffi à sacraliser l'animal puisque des gravures aussi soignées que celles de la brebis de Bou Alem et de l'ovin asexué de Daïet es-Stel représentent le sphéroïde dépourvu d'appendices; en revanche, des gravures moins soignées ou plus schématiques figurent des coiffures plus complexes. Dans de rares cas la jugulaire du sphéroïde se confond harmonieusement avec la corne (oued Nourème).

Dans les gravures schématiques le bonnet peut prendre deux formes anormales, dans le premier cas il tend à se détacher de la tête et devient une sphère tangente à l'occiput de l'animal (Oukaïmeden, Tazina, Dehar Bel Haadi), dans le second cas qui est plus fréquent le bonnet prend la forme d'une mitre plus ou moins haute (Dekhilet el-Ateuch Fedj Naam) et parfois n'est même plus séparé de la tête par un trait (Hadjar Berrik, Merdoufa), mais cette absence de séparation se remarque également sur des figures moins schématisées (El-Richa, Safiet Bou R'Nam). Pour être complet il faut signaler la 
forme particulière du bonnet à Zénaga où la brebis et le mouton asexué portent une calotte hémisphérique profondément enfoncée. Une telle forme peut être encore reconnue à Moghrar Tahtani malgré le schématisme de la gravure.

Normalement, la partie supérieure du crâne de l'ovin n'est pas visible puisqu'elle est couverte par la bonnet, ce n'est pas le cas pour les deux béliers de la grande scène mythique de Guelmouz el-Abiod dont les "sphéroïdes» et les autres éléments de parure furent rajoutés, ce qui explique également la petitesse du bonnet du mouton du registre inférieur et la disposition des gerbes piquées dans la coiffure que la proximité des pattes du bélier supérieur a obligé à disposer en deux gerbes inégales.

La parure de tête est souvent complétée par des attributs fixés au bonnet. On peut en reconnaître deux sortes : des rameaux plus ou moins courbes ou coudés ou rectilignes qui rayonnent autour de la coiffe qui sont les plus nombreux et des appendices curvilignes qui avaient été un moment qualifiés d'uraei égyptiens. Les rameaux sont disposés de manière assez anarchique, leurs dimensions dans la même gerbe peut varier. Il est exceptionnel qu'ils soient disposés symétriquement comme sur le bonnet hémisphérique du mouton asexué de Zénaga. Il arrive, surtout dans les figures schématiques ou stylisées, qu'ils forment une véritable auréole très étalée autour de la tête de l'animal (El-Krima, Aïn Ben Kerma, Merdoufa) comme autour de celle des hommes de Khrelouat Sidi Cheikh et de Moghrar Tahtani. L'aspect de ces attributs est celui de tiges assez souples et minces, comme celle du drinn (Aristida pungens), de l'alpha, ou encore de rameaux de genets. Le mouton du registre inférieur de Guelmouz el-Abiod porte sur son bonnet des attributs en lame de sabre qui ne sont pas sans rappeler les feuilles d'iris ou de glaïeul.

J'hésite à rapporter également au règne végétal les appendices curvilignes, au nombre de 2 à Bou Alem et Aïn Naga, de 3 à Zénaga, de 4 à la Gada el-Kharrouba, qui sont souvent décrits comme des plumes, bien que leur forme semble devoir se rapprocher davantage de feuilles allongées. Quoi qu'il en soit les dimensions trop réduites ne peuvent être celles de plumes d'autruche qui sont cependant les seules à présenter une telle courbure. Ces appendices uraeiformes remplacent les rameaux; à Zénaga seulement ils sont mêlés à ceux-ci. Aucun détail graphique ne permet de deviner le mode de fixation au bonnet de ces différents ornements; ils devaient être simplement piqués dans la paroi de celui-ci. Le bélier de Dakhilet el-Ateuch porte une coiffure différente de l'habituel bonnet sphérique, c'est une espèce de tiare plus haute que large dans laquelle sont plantés de part et d'autre des ornements qui semblent bien être des plumes. Cet attribut est tout à fait comparable à la coiffure que portait l'un des deux agneaux sacrifiés de la tombe 81 de la nécropole de Kerma en Nubie. 

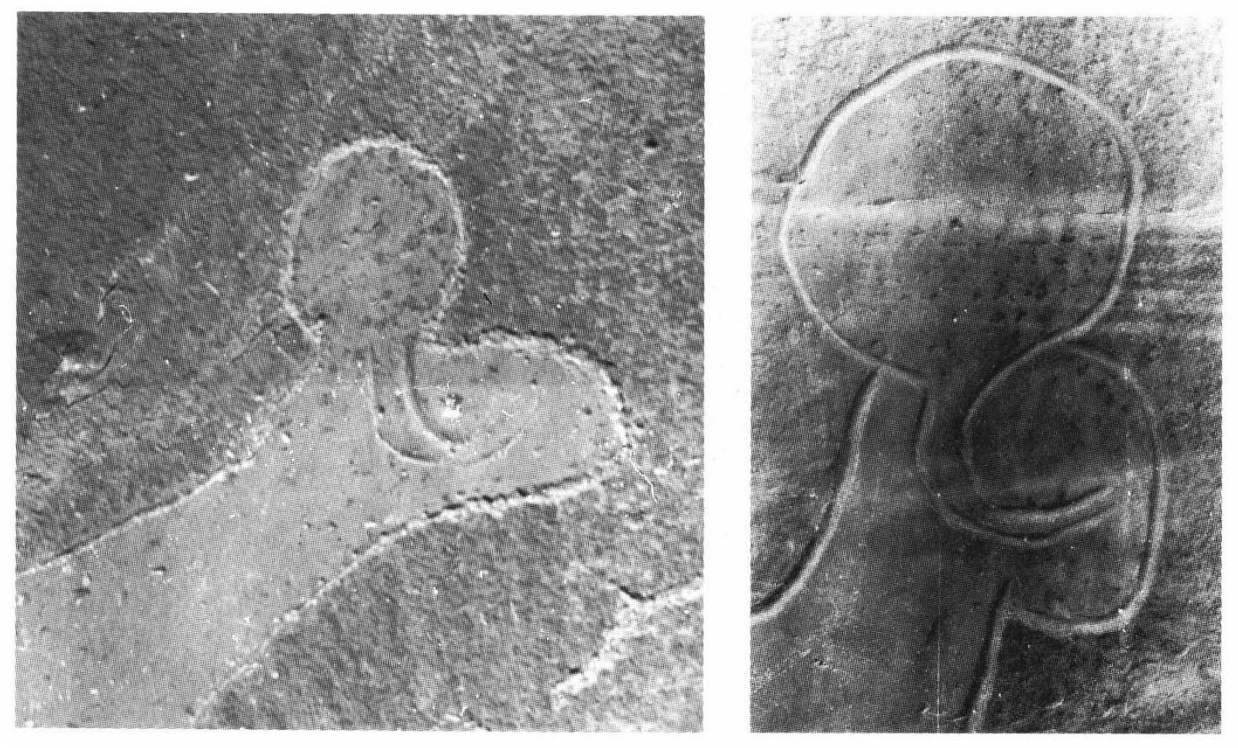

31 Il est une autre parure de tête, peu explicitée et assez mystérieuse, qui est constituée d'un ou deux éléments verticaux plantés sur le chignon de l'ovin entre deux demicercles qui, dans certaines gravures se referment pour former un disque. Dans la plupart des cas (Rosfat Men el-That, brebis de Bou Alem, Daïet es-Stel, el-Harara) ce motif n'est pas un "sphéroïde " mais des cornes en position anormale, redressées audessus de la tête comme le seraient celles de bovines ; or, les figures sont suffisamment réalistes pour que l'identification des ovins ne puisse être mise en doute. La représentation très schématique du Kef bou Beker doit être mise à part car la parure de tête semble bien être un bonnet strié de deux bandes verticales ; l'animal à garrot très prononcé et tête allongée n'est peut-être pas un ovin mais la schématisation de cette gravure est telle qu'il n'est guère possible de se prononcer.

Une autre question posée par cette série de gravures est relative à la position et la nature des demi-cercles qui remplacent le bonnet. Ce sont indubitablement des cornes dont elles ont la forme habituelle mais placées en position anormale. Cette position estelle naturelle, provoquée volontairement par les éleveurs ou ne s'agit-il que d'une liberté graphique de l'artiste ? La troisième proposition paraît très peu vraisemblable, compte tenu du réalisme général de ces gravures; il est difficile de choisir entre les deux premières. Certes quelques races de moutons ont les cornes redressées, nous avons cité le cas des moutons rackas, mais leurs cornes spiralées ne sont jamais en demi-cercle et tendent au contraire à devenir rectilignes. On connaît, en revanche, surtout dans l'Afrique orientale, de nombreux cas de déformations de cornes sur les bovins. Ces déformations figurent dans un grand nombre de stations rupestres du Tibesti et de l'Ennedi ; elles sont connues dans l'Égypte antique et sont pratiquées, de nos jours, chez les Nuer et les Dinka du Nil, chez les Souk du Lac Victoria. Mais, à notre connaissance, de telles pratiques n'ont jamais été signalées sur des ovins. Celles-ci me paraissent techniquement possibles, l'importance culturelle du bélier ou de la brebis les rendent vraisemblables. 
Aïn Naga. Le bélier de cette belle scène est bien plus grand que l'orant qui le précède. La parure du bélier est complète : sphéroïde à jugulaire, gaine de cou, collier d'épaule.

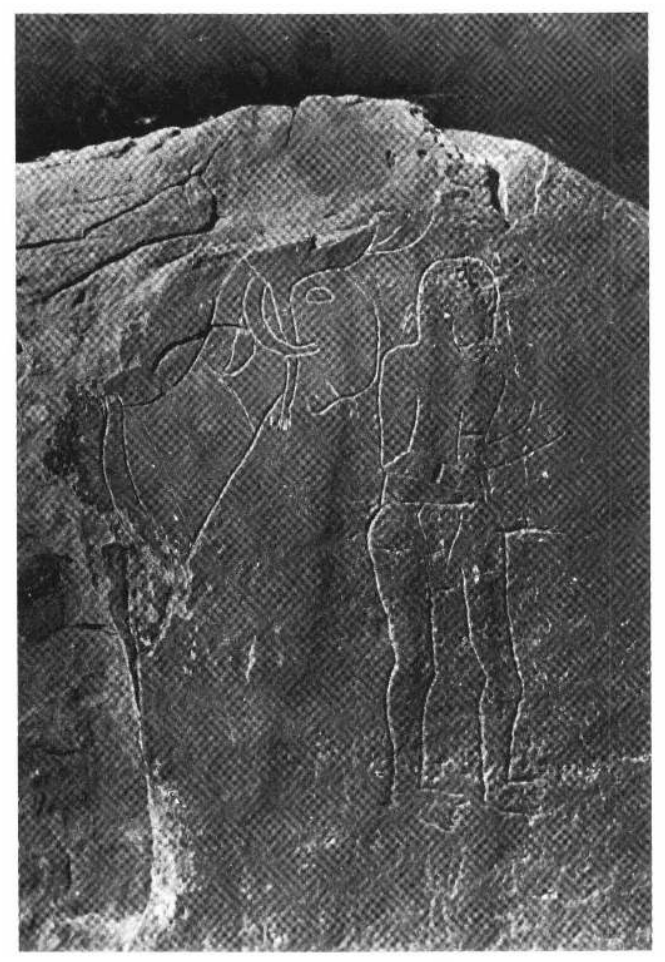

\section{La parure de cou} reposant sur les épaules tel qu'il est fidèlement représenté à Bou Alem, Aïn Naga, Zénaga, Guelmouz el-Abiod, Dekhilet el-Ateuch, Deyet el-Hamra, Hadjar Sidi Bou Beker, etc. Ce collier volumineux présente des chevrons et des motifs cloisonnés qui font penser que cette lourde parure était tressée soit en fibres végétales soit en lanières de cuir. Le collier forme un épais bourrelet qui est fort bien indiqué de part et d'autre du cou, sauf au Guelmouz el-Abiod où cette parure, comme les autres, semble avoir été rajoutée. A Khrelouat Sidi Cheikh et à Zénina ce collier descend très bas sur le poitrail tandis qu'il est dédoublé à Gada el Kharrouba, et débordant à Mokta es-Sfa.

Il n'est pas impossible que cette lourde parure ait eu une autre fonction qu'ornementale ; sa position et son poids comprimaient la trachée artère. Pour respirer l'animal était obligé de redresser le cou et de porter la tête plus relevée que d'habitude. Effectivement, les ovins porteurs de bonnet, et particulièrement ceux qui ont ce collier, ont la tête redressée, le cou formant avec l'axe du corps un angle de 120 à $130^{\circ}$ alors que dans le port naturel le cou et la tête sont pratiquement dans le prolongement de cet axe.

Cet artifice destinée à donner à l'animal un port altier me semble, dans deux cas au moins, complété par un dispositif comprimant. En examinant les deux gravures de Bou Alem et d'Aïn Naga qui sont les plus précises et détaillées on remarque le long du cou, entre l'extrémité tombant de l'oreille et le collier d'épaule, une zone délimitée par un ou deux traits. A Bou Alem cette partie du cou est entièrement polie et dépourvue de poils ; il s'agit vraisemblablement d'une sorte de gaine en étoffe ou en cuir souple qui

Encyclopédie berbère, 9 | 1991 
comprime le cou et ne porte aucun ornement. On pourrait y voir aussi une zone rasée dont on ne comprend guère la raison d'être. L'égorgement du mouton se faisant plus haut, juste sous la mandibule, le rasage de cette région du cou ne pourrait même pas être considérée comme une préparation du sacrifice.

D'autres ovins munis de sphéroïde portent un collier plus simple sur la partie médiane du cou; il se distingue à la fois du gros collier d'épaule tressé et de la gaine de cuir signalée ci-dessus. Ces colliers ont toujours un certain volume et sont parfois assez larges (El-Harra, Dehar Bel Haadi, Rcheg Dirhem). A Hadjerat Sidi Bou Beker le collier présente des incisions verticales qui suggèrent une segmentation, perles ou motifs articulés.

\section{La parure d'échine}

Elle n'est reconnaissable que sur les gravures de Bou Alem et de Guelmouz el-Abiod; compte tenu de l'identité des parures on peut supposer qu'elle existait aussi à Aïn Naga mais la roche est malheureusement desquamée immédiatement en arrière du collier. Cette parure est rendue sur les gravures par une ligne festonnée intérieure parallèle à la ligne du garrot et de l'échine, elle semble s'arrêter au point le plus saillant de la croupe. Il ne s'agit pas d'une représentation stylisée de la courte crinière que possèdent les béliers des diverses races de moutons à poils car le pelage est traité d'une manière très réaliste à Bou Alem; de plus la crinière aurait été représentée au-dessus de la ligne du dos et non à l'intérieur du corps. Il s'agit donc d'une bande assez étroite, découpée dans un matériau souple et posée sur l'échine de l'animal. Cette parure, vraisemblablement en cuir, était trop étroite pour tenir toute seule sur le dos, or elle est étroitement appliquée comme si elle était tendue. Seul un dispositif, invisible sur les gravures, comme par exemple une croupière passant sous la naissance de la queue pouvait assurer un tel maintien. Il est possible que ce "caparaçon" formât avec le collier d'épaule une sorte de harnais.

\section{Relations entre les ovins à sphéroïde et les hommes}

L'intérêt des représentations de "béliers à sphéroïde » ne réside pas seulement dans la figuration de l'animal et de ses parures diverses. Cette figure appartient parfois à des scènes complexes dans lesquelles entrent des personnages. Ces relations entre l'homme et le bélier ne sont pas toujours très claires dans ces scènes. Les figurations humaines les plus belles sont celles de Gada el-Kharrouba, d'Aïn Naga, de Bou Alem et de Dakhilet el-Ateuch; dans ces scènes, l'homme précède le bélier, lui tournant le dos et s'avance en position d'orant. A Ksar el Ahmar la situation est semblable bien que l'homme soit armé d'une hache et que le mouton asexué soit dépourvu de toute parure. A Bou Alem l'homme, moins profondément gravé que le bélier, porte un bouclier ou plus vraisemblablement un sac ou un carquois comme les personnages de l'Oued Dermel et de tant d'autres gravures. Dans la scène complexe de Guelmouz el-Abiod, un personnage filiforme s'intercale entre l'animal plus ou moins mythique et ouvert en deux et le plus grand des béliers. Il a une attitude d'orant, jambes et bras à demi fléchis et il est vu de face. A Moghrar Tahtani, autre scène mythique, l'ovin à sphéroïde est juché sur un personnage de style macaronique dont la tête s'engage entre les pattes de 
l'animal, ne faisant qu'un avec lui; devant lui et lui tournant le dos s'avance un personnage en position d'orant et portant la même coiffure rayonnante.

Gada el-Kharrouba (calque sur photo très oblique de R. Vaufrey). Scène identique à celle d'Aïn Naga.

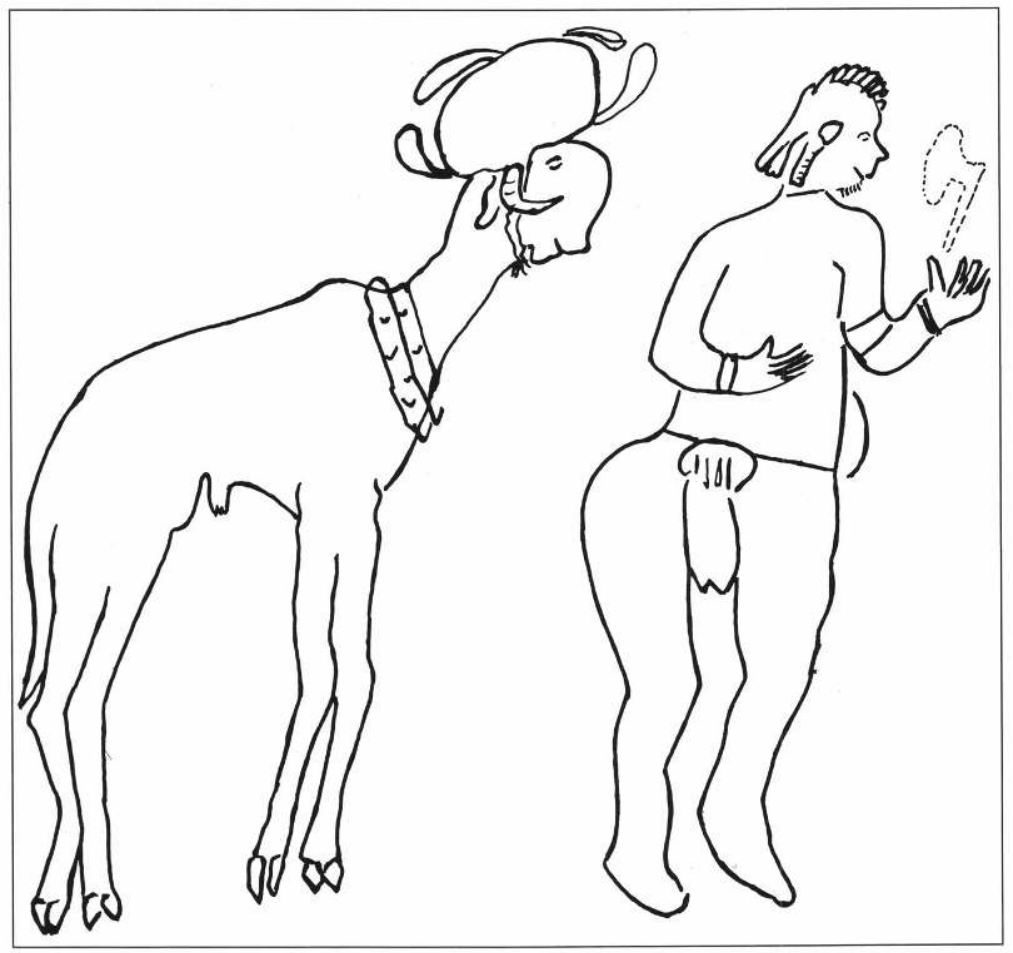

Si cette disposition du couple homme-bélier est la plus fréquente elle n'est pas la seule ; il arrive en effet que l'ovin et l'homme soient affrontés comme à Daïet es-Stel, Safiet Larba, Hadjar Berrik et El-Harara. Il faut tenir compte aussi de la position frontale de l'homme comme à Guelmouz el-Abiod déjà mentionné ou à El-Richa.

L'homme est toujours placé à droite du bélier sauf dans trois cas : Daïet es-Stel, Hadjar Berrik et Oukaïmeden.

Les tailles réciproques de l'homme et du bélier ne sont pas toujours respectées, dans six cas au moins, le bélier est plus grand que le personnage, ailleurs ils sont de tailles égales; il arrive enfin qu'un personnage minuscule soit ajouté après coup (El-Hasbaya, Kef Bou Beker). Les hommes sont assez souvent armés, dans un cas (Gada el-Kharrouba) une hache a été ajoutée au-dessus de la main de l'homme; mais une fois seulement l'arme semble vraiment dirigée contre le bélier; c'est à El-Harara où précisément les ovins présentent une anomalie de cornage. Nous avons déjà signalé la scène de Rosfat Men el-That où l'archer est placé entre deux moutons qui présentent la même anomalie. Dans les autres cas, les personnages armés tournent le dos au bélier. 


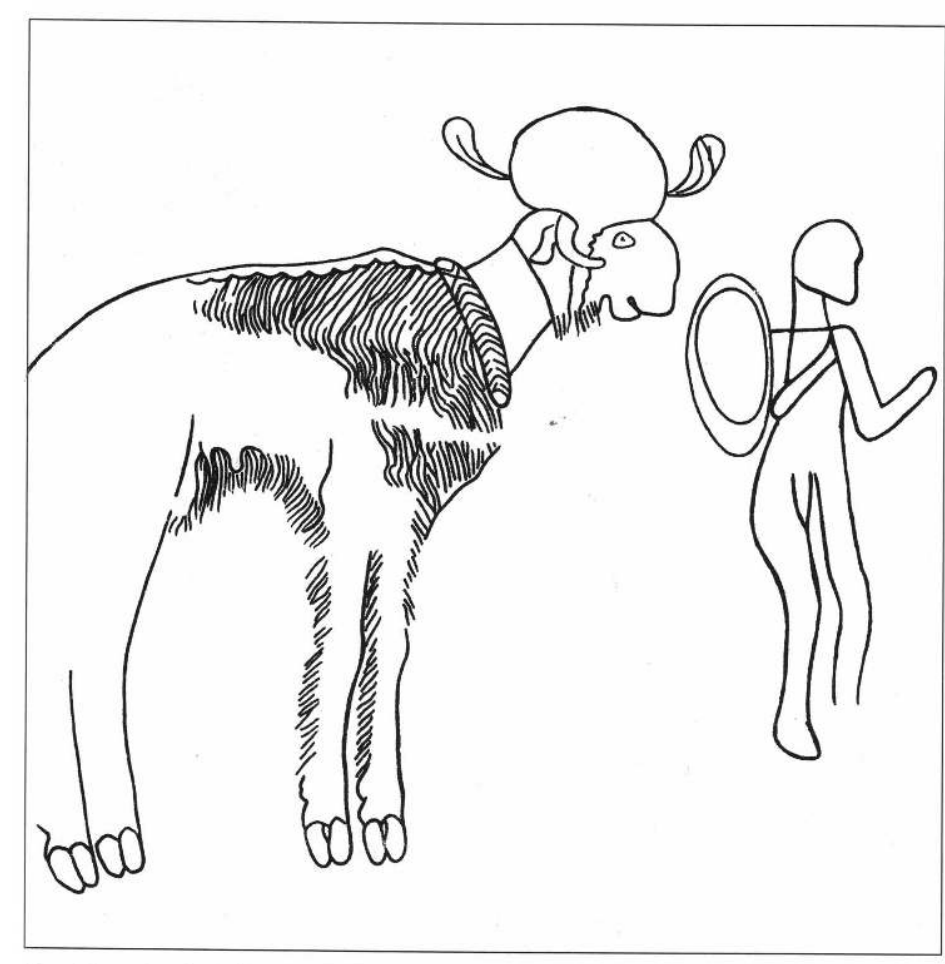

Le bélier porte la parure complète : sphéroïde à appendices uraeiformes, jugulaire festonnée, gaine de cou, collier tressé et « caparaçon ».

La position classique est donc celle de l'orant placé à droite du bélier, lui tournant le dos ou vu de face. L'attitude même de ces orants est sujette à quelques variations. Les plus précises est les plus soignées des gravures (Aïn Naga, Gada El-Kharrouba, Bou Alem, Daïet es-Stel, Dakhilet el-Ateuch) montrent les hommes de trois-quarts, les bras collé $s$ au corps, les avant-bras à demi relevés. Dans les cinq scènes, pourtant fort éloignées les unes des autres, l'avant-bras gauche est légèrement plus relevé que le droit et les hommes portent à Aïn Naga et à Gada El-Kharrouba le même cache-sexe, simple bande d'étoffe ou de cuir retenue à un lien qui passe autour de la taille. L'extrémité retombe par devant et est fixée par des boutons circulaires à Aïn Naga, des bâtonnets allongés ou des coutures à Gada el-Kharrouba. L'extraordinaire ressemblance entre ces deux gravures est encore accentuée par le port d'un bracelet au poignet droit, complété à Aïn Naga par un bracelet de biceps à gauche. Cette ressemblance permet, à mon avis, de régler définitivement la question relative à la hache piquetée au-dessus de la main gauche de l'homme de Gada el-Kharrouba : ce ne peut être qu'un rajout car elle ne figure pas sur la scène identique d'Aïn Naga.

Dans d'autres scènes les personnages qui sont de style négligé (Guelmouz, el-Richa, ElHadj Mimoun, El-Harara, Rosfat) sont nus et parfois ithyphalliques. Une troisième catégorie est constituée par des porteurs de pagne (Moghrar Tahtani, Feidj Naam) ou de vêtements plus complexes (Hadjar Berrik) ; or, toutes ces gravures appartiennent à un style différent de celui des grandes gravures naturalistes duquel dépendant les plus belles scènes. Des observations complémentaires peuvent être faites sur les coiffures et les masques des hommes associés aux béliers à sphéroïde. 


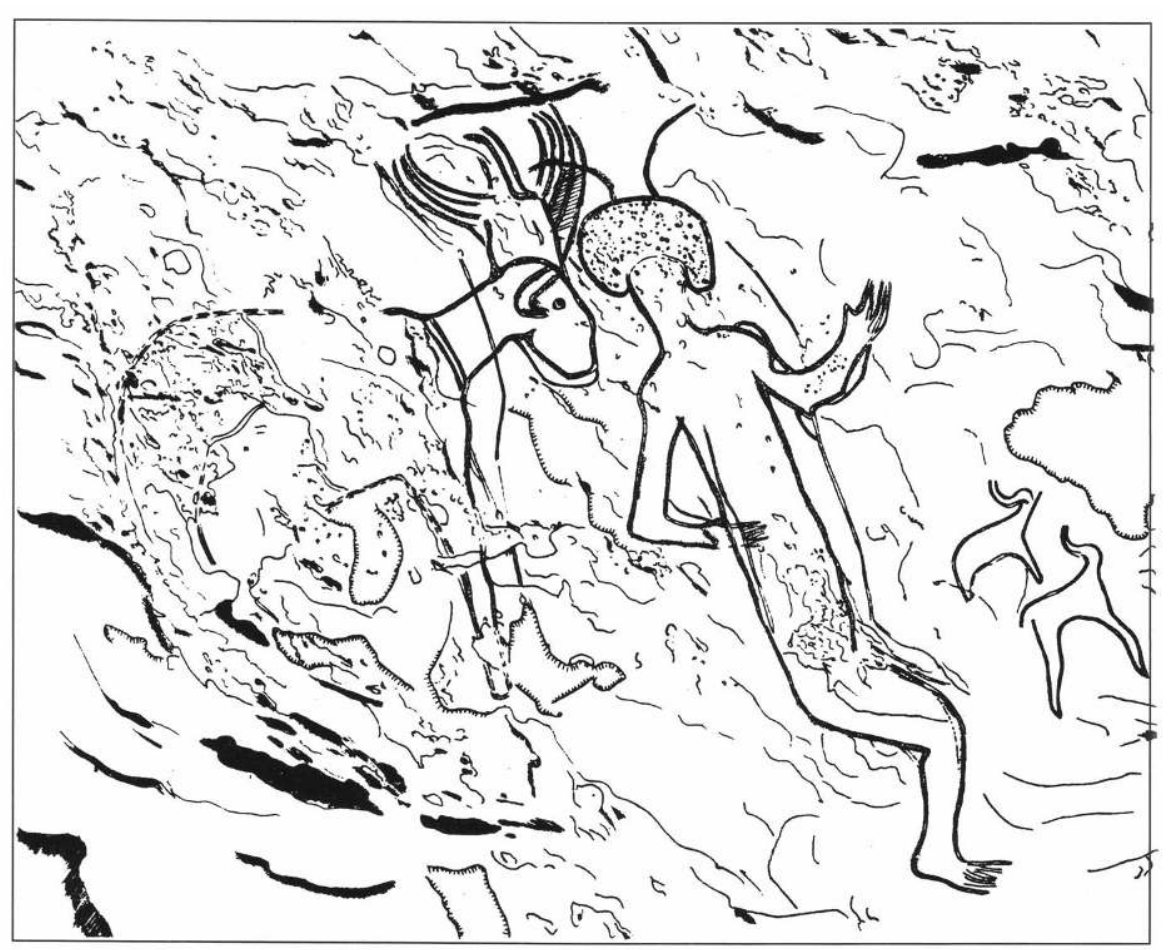

Scène identique à celles d'Aïn Naga, Gada el-Kharrouba et Bou Alem. Le bélier porte une tiare ornée de plumes d'autruche et l'orant qui le précède porte lui-même un sphéroïde à antennes divergentes (relevé F. Soleilhavoup).

Les sujets masqués sont au nombre de deux : à Khrelouat Sidi Cheikh, le personnage porte un masque dont l'intérieur est entièrement piqueté pour souligner sa nature artificielle. Le masque couvre la tête et le cou, son profil est celui d'un ovin. L'homme est placé sous le mufle du bélier. Du masque s'échappent des rameaux qui rayonnent en deux gerbes de part et d'autre du museau du bélier. A Feidj Naam, le personnage qui est très stylisé porte, semble-t-il un masque, mais, à en juger par l'attitude du corps, le museau serait situé en arrière.

Plus intéressante est la coiffure de deux des trois personnages qui à Moghrar Tahtani sont en relation avec le bélier à sphéroïde, le personnage de style macaronique, d'où semble s'extraire l'ovin, a une tête globuleuse radiée et les rayons apparaissent en surimpression sur le corps de l'animal ; l'orant qui les précède a une coiffure ou plus exactement un masque rayonnant du même type. A Dakhilet el-Ateuch le personnage porte une coiffure sphérique surmontée de deux antennes courbes divergentes.

A el-Harara et Hadjar Berrik, l'homme qui fait face au bélier porte une sorte de chapeau à trois protubérances dont $\mathrm{H}$. Lhote a montré qu'il n'était pas exceptionnel dans l'art rupestre nord-africain; les détails qui remplissent l'intérieur de cette figuration à Hadjar Berrik font penser qu'il s'agit d'un chapeau de paille. Chose curieuse, à Daïet esStel, l'homme qui fait également face au bélier, semble porter une coiffure qui serait une sorte de calotte. 


\section{Conclusion}

L'examen des relations entre l'homme et le bélier à sphéroïde permettent, sinon de résoudre toutes les questions, du moins de suggérer quelques interprétations.

En haut - scènes schématiques de Merdoufa (sphéroïde stylisé, personnage peut-être plus récent) et de Fedj Naam (ovin portant une tiare, personnage masqué et armé portant un pagnen à queue postiche).

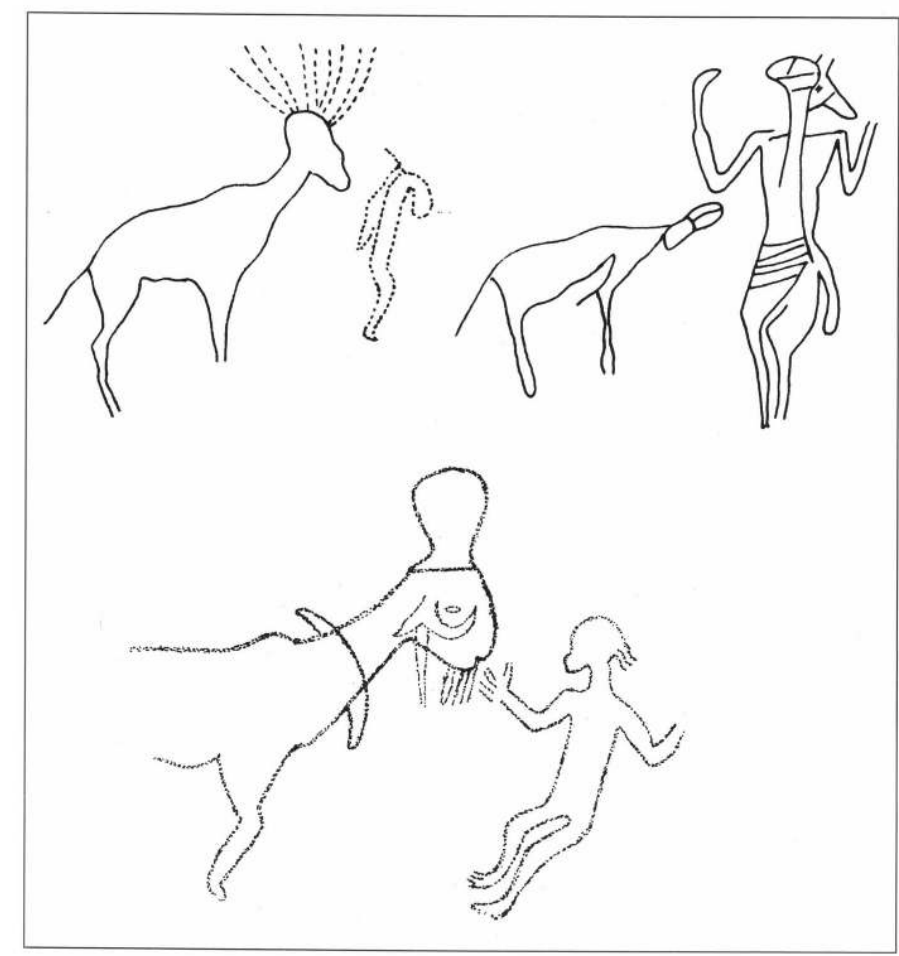

En bas - Merdoufa : bélier et orant lui faisant face ; la jugulaire est dissociée du sphéroïde qui semble ici être une calebasse.

Les plus importantes représentations, soit par leur complexité (Moghrar Tahtani, Guelmouz el-Abiod) soit par leur précision documentaire (Aïn Naga, Gada el-Kharrouba, Bou Alem, Khrelouat Sidi Cheikh Dakhilet el-Ateuch) ne permettent aucunement de parler d'un culte du bélier, car l'orant tourne le dos, on ne fait pas face à l'animal. Dans les rares cas où cette position affrontée est reproduite (Daïet es-Stel, Feidj Naam, Hadjar Berrik, El Harara), l'homme porte une coiffure ou un masque et dans deux cas est armé d'une hache ; le bélier porte un sphéroïde simple ou des cornes relevées. Ces scènes semblent donc correspondre soit à une autre phase du cérémonial, soit à une transformation des croyances, à une époque plus récente. Quoi qu'il en soit, l'examen sans idée préconçue de ces différentes scènes ne permet pas d'affirmer l'existence d'un culte du bélier.

La précision des attributs dans les œuvres majeures montre bien que ces béliers sont des animaux parés suivant un rituel assez précis et destiné vraisemblablement à être sacrifiés à la fin d'une cérémonie. Pour donner plus de prestance à l'animal, un agencement a été conçu pour l'obliger à redresser la tête. Sa parure de tête et souvent son gigantisme par rapport à l'homme ont pour but de valoriser la victime offerte à la 
divinité. Les gravures étaient peut-être destinées à matérialiser le sacrifice et à perpétuer son souvenir et donc son efficacité.

Si cette hypothèse était acceptée on pourrait s'étonner que la divinité, ou ce qui en tient lieu dans les croyances des Néolithiques de l'Atlas, n'ait pas été figurée, mais il n'est pas sûr que ces premiers pasteurs aient ressenti le besoin de représenter le dieu, ni même qu'ils aient été capables de la concevoir sous des traits susceptibles d'être reproduits.

\section{BIBLIOGRAPHIE}

ALIMEN H., La station rupestre de Marhouma (Sahara occidental), Institut de recherches sahariennes, Alger, 1954.

CADENAT P., « Les gravures rupestres des environs de Tiaret (Départ. d'Oran) », Actes du II Congr. panafric. de Préhist., Alger, 1952 (1955), p. 701-713.

CAMPS G., Les civilisations préhistoriques de l'Afrique du Nord et du Sahara, Paris, Doin, 1974.

CAMPS G., « Origines de la domestication en Afrique du Nord et au Sahara », Rev. franc. d'Hist.

d'Outre-mer, t. LXV, p. 363-376.

CAMPS G., « Un thème religieux dans l'art rupestre nord-africain. Le bélier à sphéroïde », in Studi in onore di Salvatore Puglisi, Rome, 1985, p. 345-357.

CAMPS G., « Les croyances protohistoriques en Afrique du Nord », in Mythes et croyances du monde entier, Lidis, Paris, 1985, t. 2, p. 304-319.

CAMPS G., « Scènes de caractère religieux dans l'art rupestre de l'Afrique du Nord et duSahara », in Mélanges Pierre Lévêque, Besançon, 1988, p. 65-82.

CURASSON C, Le mouton du Soudan français, Paris, Union ovine colon. S.D.

DOUTRESSOULLE G., L'élevage du Niger, thèse pour le doctorat vétérinaire, Mortain, 1924.

DOUTRESSOULLE G., L'élevage au Soudan français, Alger, 1952.

FLAMAND G.-B., Les Pierres écrites, Paris, Masson, 1924.

ESPÉRANDIEU G., « Domestication et élevage dans le Nord de l'Afrique au Néolithique et dans la Protohistoire d'après les figurations rupestres ", Actes du II ${ }^{e}$ Congr. panafric. de Préhist., Alger, 1952 (1955), p. 551-573.

FROBENIUS L. et OBERMAIER H., Hadschra Maktouba, Munich, Wolff, 1925.

GAILLARD Cl., «Le bélier de Mendès ou le mouton domestique de l'Ancienne Égypte », Bull. de la Soc. d'Anthrop. de Lyon, séance du 4 mai 1901, p. 69-95.

GSELL St., Monuments antiques de l'Algérie, Paris, 1901, p. 46 ; Histoire ancienne de l'Afrique du Nord, t. I, Paris, 1927, p. 250-253. 
JOLEAUD L., " Gravures rupestres et rites de l'eau », Journal de la Soc. des Africanistes, t. III, 1933, p. 197-282 et t. IV, 1934, p. 285-302.

LEFEBVRE G., « La station de gravures rupestres de Daïet es-Stel (Sud algérois) », Libyca, t. XV, 1967, p. 207-213.

LETHIELLEUX J., « Vestiges préhistoriques et protohistoriques de la région de Djelfa », Libyca, t. XIII, 1965 , p. 249-265.

LHOTE H., Les gravures rupestres du Sud-Oranais, C.R.A.P.E., XVI, Paris, AMG, 1970.

LIBMANN P., Le mouton dans les gravures et les peintures rupestres de l'Afrique du Nord et du Sahara, thèse pour le doctorat vétérinaire, Toulouse, 1979.

LHOTE H., Les gravures rupestres de l'Atlas saharien. Monts des Ksour Ouled Nail et région de Djelfa, Alger, 1984.

SOLEILHAVOUP F., « Les surfaces de l'Art rupestre en plain air : relations avec le milieu biophysique et méthode d'étude ", L'anthropologie, t. XI, 1986, p. 743-782.

VAUFREY R., L'Art rupestre nord-africain, Inst. de Paléontologie Humaine, Mém., 20, 1939.

\section{INDEX}

Mots-clés : Zoologie, Art rupestre, Préhistoire 